\title{
A INFLUÊNCIA DA ESPIRITUALIDADE/RELIGIOSIDADE NO ENFRENTAMENTO DO CÂNCER DE MAMA: UMA REVISÃO INTEGRATIVA
}

\author{
The influence of spirituality/religiosity coping with breast cancer: an integrative \\ review
}

Matheus Nepomuceno de Araújo ${ }^{1}$ Gislaine de Souza Ferreira Araujo ${ }^{2}$ (D) Marcio Martins da Costa ${ }^{1}$ Caren Camargo do Espírito Santo ${ }^{3}$ (D) Ana Paula Munhen Pontes ${ }^{1}$

${ }^{1}$ Centro Universitário de Valença (UNIFAA) Valença $(R J)$

${ }^{2}$ Maternidade Escola de Valença - Valença (RJ)

${ }^{3}$ Centro Universitário Gama e Souza (UNIGAMA) - Rio de Janeiro (RJ)

Autor correspondente:

Ana Paula Munhen Pontes

E-mail: ana.munhen@faa.edu.br

\section{Como citar este artigo:}

ARAÚJO, M. N.; ARAÚJO, G.S.F.; COSTA, M.M.; ESPÍRITO SANTO, C.C.; PONTES, A. P. M. A influência da espiritualidade/religiosidade no enfrentamento do câncer de mama: uma revisão integrativa. Revista Saber Digital, v. 14, n. 3, p. 08-22, 2021.

Data de Submissão: $31 / 08 / 21$

Data de aprovação: 26/09/21

Data de publicação: 21/12/21

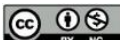

Esta obra está licenciada com uma licença http://creativecommons.org/licenses/by-nc/4.0/

\section{RESUMO}

Objetivo: Esta pesquisa objetivou analisar as evidências presentes na literatura nacional acerca da influência da espiritualidade/religiosidade no enfretamento do câncer de mama. Método: Trata-se de uma revisão integrativa da literatura que consiste na análise e síntese de estudos publicados que contribuem para melhor compreensão e aprofundamento do tema investigado. Resultados: Foram selecionados 12 (doze) artigos que se adequaram aos objetivos do trabalho. Os artigos selecionados no estudo de acordo com ano, título, autores, periódico, região de realização e categoria profissional do autor principal foram colocados em um quadro para melhor síntese dos estudos. Para melhor expor a análise, interpretação e discussão dos artigos, optou-se pela divisão em categorias, sendo elas: A espiritualidade no enfrentamento do câncer de mama: aceitação, resiliência e conforto; A espiritualidade/religiosidade como rede de apoio social no enfrentamento do câncer de mama; A dimensão espiritual como instrumento de prática profissional no cuidado holístico a mulheres com câncer de mama. Conclusão: A espiritualidade/religiosidade representada por Deus e pela fé se apresenta como uma estratégia extremamente importante no enfrentamento do câncer de mama, promovendo mais conforto e alívio dos impactos causados pela doença, além de bem-estar e resiliência. Conhecer e valorizar a vivência espiritual destas pacientes torna-se então muito importante, inclusive pelos profissionais que prestam cuidados diretamente a elas, uma vez que, contribuem para o bem-estar e sensação de apoio e assistência.

Palavras-chave: Câncer de mama, Enfermagem, Espiritualidade.

\section{ABSTRACT}

Objective: This research aimed to analyze the evidence present in the national literature about the influence of spirituality/religiosity on breast cancer control. Method: This is an integrative review of the literature that consists in the analysis and synthesis of published studies that contribute to a better understanding and deepening of the theme investigated. Results: Twelve (12) articles that fit the objectives of the study were selected, the articles selected in the study according to year, title, authors, journal, region of achievement and professional category of the main author were placed in a table for better synthesis of the studies. To better expose, the analysis and interpretation and discussion of the articles, we opted for the division into categories, which are: Spirituality in coping with breast cancer: acceptance, resilience and comfort; Spirituality/religiosity as a social support network in coping with breast cancer; The spiritual dimension as an instrument of professional practice in holistic care for women with breast cancer. Conclusion: The spirituality/religiosity represented by God and faith are an extremely important strategy in coping with breast cancer, promoting more comfort and relief from the impacts caused by the disease, as well as well-being and resilience. To give birth to and value the spiritual experience of these patients, it is then very important, including for professionals who provide care directly to them, since they contribute to the well-being and feeling of support and care.

Keywords: Breast cancer, Nursing, Spirituality. 


\section{INTRODUÇÃO}

Segundo a OMS (Organização Mundial da Saúde), em 2018, o câncer de mama foi diagnosticado em 2,09 milhões de pessoas no mundo. Já no Brasil, segundo dados do INCA (Instituto Nacional do Câncer) foram totalizados 59.700 $(29,5 \%)$ novos casos de câncer no país como um todo, e $74,67 \%$ dos casos de câncer no Rio de Janeiro (INCA, 2019).

Segundo Caetano e Soares (2005, p. 210) "o câncer possui caráter estigmatizante, é visto como sinônimo de morte e ocasiona transformações dolorosas na vida das mulheres". Ao identificar que essa situação agride a mulher de forma emocional e física é necessário que os profissionais de saúde e toda rede de apoio busquem ao máximo auxiliá-las a alcançar uma melhor qualidade de vida.

Diante de um cenário tão devastador, quando se recebe um diagnóstico positivo para câncer, a espiritualidade, que é considerada pela OMS como uma das dimensões da qualidade de vida, emerge (AITKEN, 2012).

A espiritualidade "move-se para além da ciência e da religião instituída. Ela é considerada mais primordial, mais pura e mais diretamente relacionada com a alma em sua relação com o divino" (SAPORETTI; SILVA, 2012, p.558). Já religião surge constantemente associada à instituição religiosa, organizada, que possui dogmas a serem seguidos e cerimônias (AITKEN, 2012). "A religião instituída (...) pertence à dimensão cultural e social e pode ser considerada espiritual se realmente relaciona o indivíduo com o seu sagrado ou transcendente. Cada religião expressa o espiritual de um povo conforme suas características sociais e culturais" (SAPORETTI; SILVA, 2012, p.557).

Desse modo, a religião pode ser definida como um sistema que envolve crenças, práticas e rituais relacionados ao transcendente, ao mesmo tempo em que abarca um conjunto de crenças acerca da vida após a morte e de papéis sociais e culturais dentro de um grupo sócio-religioso. Já a espiritualidade é 
concebida como uma relação com o sagrado e o transcendente, no domínio do espírito, do extra-físico e do não-material (KOENIG, 2012).

A espiritualidade tende a ser algo mais intenso, individual e espontâneo associado a sentimentos de amor, profundidade, mistérios e inspiração (AITKEN, 2012). Esta mesma autora afirma que "crenças religiosas estão relacionadas com melhor saúde e maior qualidade de vida (AITKEN, 2012, p. 365).

Partindo desse ponto definiu-se como questão de pesquisa: Qual a influência da espiritualidade no enfrentamento do câncer de mama? Esta temática apresenta-se com grande relevância uma vez que estudos apontam a necessidade de maiores investigações, como o realizado por Mesquita et al. (2013), que identificou que pacientes com câncer consideraram importante a espiritualidade/religião em suas vidas e estariam satisfeitos no recebimento de um cuidado espiritual.

A motivação surgiu da intenção dos autores em saber como espiritualidade influencia na qualidade de vida das pessoas no enfrentamento da moléstia, devido ao potencial de sua malignidade e nas mudanças que a mesma acarreta no cotidiano das pessoas acometidas. Nesta pesquisa foi utilizada a expressão espiritualidade/religiosidade, considerando a religiosidade como uma dimensão da espiritualidade.

Neste sentido, este estudo possui como objetivo analisar as evidências presentes na literatura nacional acerca da influência da espiritualidade/religiosidade no enfrentamento do câncer de mama.

\section{MATERIAIS E MÉTODOS}

Trata-se de um estudo de Revisão Integrativa da Literatura, que consiste na análise e síntese de estudos publicados que contribuem para melhor compreensão e aprofundamento do tema investigado. Essa metodologia tem sido uma ferramenta importante na área da saúde, pois permite organizar e sintetizar as pesquisas disponíveis sobre determinado tema, analisá-las, identificar lacunas no conhecimento e, dessa forma, auxiliar na melhora da 
prática clínica fundamentada no conhecimento científico, através da comparação entre diversos estudos (MENDES; SILVEIRA; GALVÃO, 2008).

Para a sua formulação é necessário transcorrer seis etapas distintas, conforme descrita por Ganong (1987) apud Souza; Silva e Carvalho (2010). Primeira etapa- A formulação da pergunta norteadora; segunda etapa - Busca na literatura; terceira etapa - Categorização dos estudos; quarta etapa Avaliação dos estudos incluídos na revisão; quinta etapa - Interpretação dos resultados e sexta etapa - Síntese do conhecimento.

Atendendo a primeira etapa foi definida como pergunta norteadora: quais as evidências presentes na literatura brasileira acerca da influência da espiritualidade no enfrentamento do câncer de mama?

As fontes de dados para a pesquisa foram a Literatura Latina Americana e do Caribe em Ciências em Saúde (LILACS), Medical Literature Analysis and Retrieval System Online (MedLine), Base de Dados em Enfermagem (BDENF) e Scientific Eletronic Library Online (SCIELO). A busca foi realizada em julho e agosto de 2020, utilizando os seguintes Descritores em Ciências da Saúde (DECS): espiritualidade and câncer de mama; espiritualidade and câncer de mama and enfermagem.

Como critérios de inclusão foram utilizados artigos com textos completos, publicados em português, no período de 2015 a 2020. Foram excluídos teses, dissertações, textos em língua estrangeira, textos duplicados nas bases de dados e todos os artigos que não se adequavam ao tema da pesquisa.

A partir da busca realizada foram encontradas 1810 publicações, sendo excluídos 1709 após aplicação dos critérios de inclusão e exclusão. Do material pré-selecionado, 101 artigos, procedeu-se a leitura minuciosa de cada resumo/artigo incluindo aqueles que se adequavam à temática e ao objetivo propostos. Desta forma, seguindo todos os critérios descritos foram selecionados 12 artigos para análise (Figura 1). 
Figura 1 - Diagrama de seleção dos artigos. Valença/RJ, Brasil, 2020.

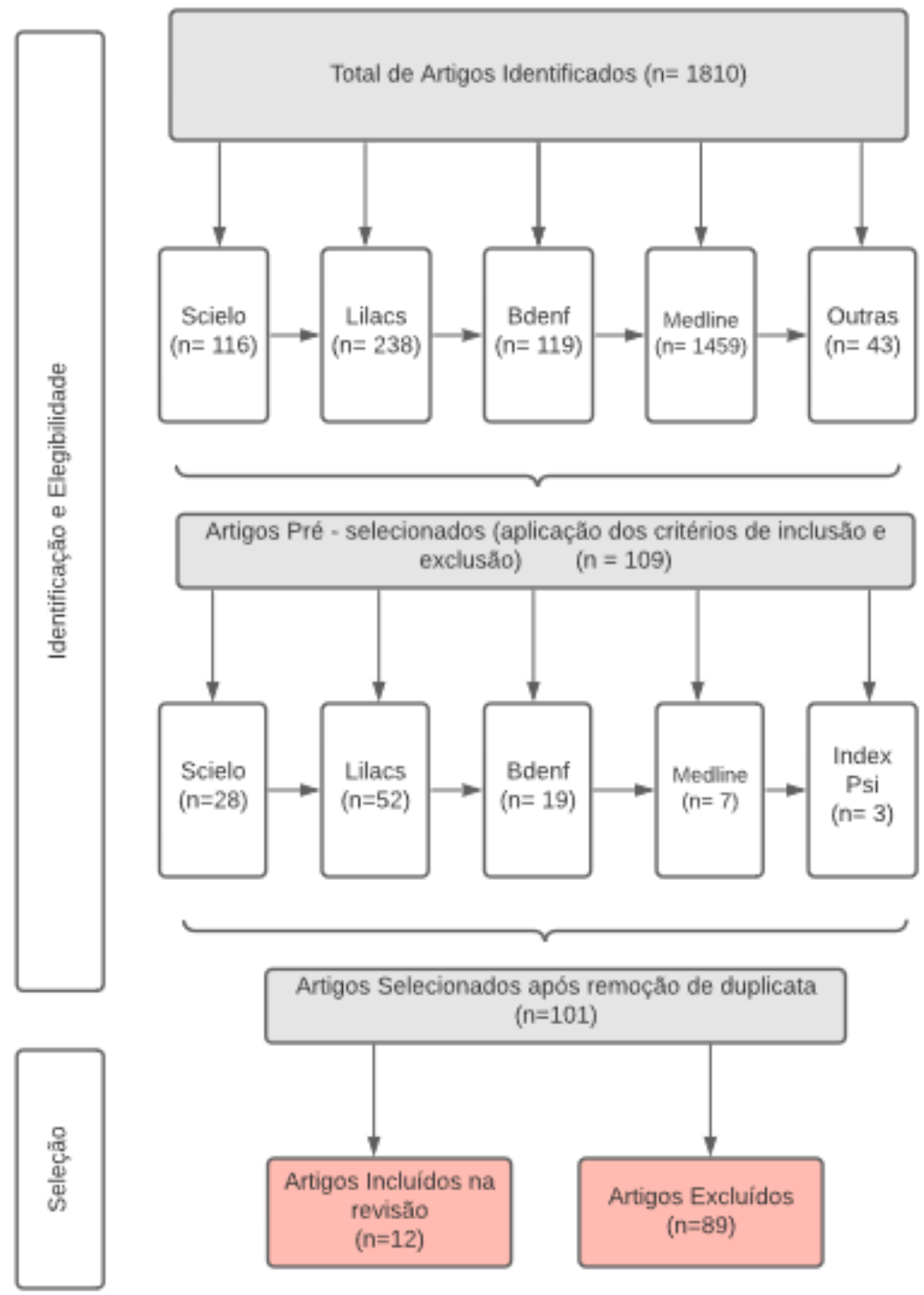

Fonte: autores.

Atendendo a terceira etapa, para a organização e tabulação dos dados, foi elaborado, pelos autores, um instrumento de coleta de dados em forma de tabela com auxílio do software Excel, contendo: ano, título do artigo, autores, periódico, objetivo, natureza do estudo, principais resultados, região de realização do estudo, e categoria profissional do primeiro autor. 
Em sequência, na quarta etapa, foi realizada uma análise bibliométrica para caracterização dos estudos selecionados e realizada a análise crítica, os trabalhos foram agrupados e comparados por conteúdos similares, com a construção de três categorias, intituladas: A espiritualidade no enfrentamento do câncer de mama: Aceitação, Resiliência e Conforto; A espiritualidade/religiosidade como rede de apoio social no enfrentamento do Câncer de mama; A dimensão espiritual como instrumento de prática profissional no cuidado holístico a mulheres com câncer de mama.

$\mathrm{Na}$ quinta etapa, referente interpretação dos resultados, foi possível identificar possíveis lacunas de conhecimentos e prioridades para estudos futuros. E finalmente na sexta etapa, parte da finalização, ocorreu a apresentação da revisão de forma clara e completa, discussão dos resultados e possíveis propostas para uma futura continuidade da pesquisa. Por tratar-se de uma revisão bibliográfica este estudo não necessita da aprovação do Comitê de Ética em Pesquisa para ser desenvolvido.

\section{RESULTADOS}

Foram selecionados 12 (doze) artigos que se adequaram aos objetivos do trabalho. O quadro a seguir apresenta a síntese dos estudos. Em relação ao ano de publicação, 2 (16,6\%) artigos foram publicados em 2015; 2 (16,6\%) em 2017; $2(16,6 \%)$ em 2018; 4 (33,6\%) em 2019, totalizando o ano de maior publicação até o momento. E, finalmente, 2020 com duas (16,6\%) publicações sobre o tema. No ano de 2016 não houve nenhum artigo selecionado.

Quanto à área de atuação dos autores dos artigos $10(83,3 \%)$ são enfermeiros, divididos em graduandos e mestrandos; 1 (8,3\%) graduanda em fisioterapia, e por fim 1 (8,3\%) graduanda em psicologia.

As regiões geográficas de realização dos artigos são, da maior publicação para menor: Nordeste 50\% (6), Sul 25\% (3), Sudeste 16,3\% (2) e Europa com $8,3 \%(1)$. 
Quadro 1 - Síntese e caracterização dos estudos selecionados para análise.

Valença/RJ, 2021.

\begin{tabular}{|c|c|c|c|c|c|}
\hline Ano & Título do artigo & Autores & Revista & Região & $\begin{array}{l}\text { Categoria } \\
\text { Profissional } \\
\text { do primeiro } \\
\text { autor }\end{array}$ \\
\hline 2015 & $\begin{array}{l}\text { Sentimentos e fontes } \\
\text { de apoio emocional de } \\
\text { mulheres em pré- } \\
\text { operatório de } \\
\text { mastectomia em um } \\
\text { hospital-escola }\end{array}$ & $\begin{array}{c}\text { NASCIMENTO } \\
\text {, K.T.S et al. }\end{array}$ & $\begin{array}{l}\text { Revista de } \\
\text { enfermagem } \\
\text { UERJ }\end{array}$ & Nordeste & Enfermeira \\
\hline 2015 & $\begin{array}{c}\text { Preditores da } \\
\text { qualidade de vida } \\
\text { numa amostra de } \\
\text { mulheres com cancro } \\
\text { da mama }\end{array}$ & $\begin{array}{l}\text { SOUSA, H.; } \\
\text { GUERRA, M.; } \\
\text { LEONOR, L. }\end{array}$ & $\begin{array}{c}\text { Análise } \\
\text { Psicológica }\end{array}$ & Portugal & $\begin{array}{l}\text { Graduanda } \\
\text { em } \\
\text { Psicologia }\end{array}$ \\
\hline 2017 & $\begin{array}{c}\text { Câncer de mama: o } \\
\text { apoio recebido no } \\
\text { enfrentamento da } \\
\text { doença }\end{array}$ & $\begin{array}{l}\text { SANTOS, } \\
\text { I.D.L. } \\
\text { et al. }\end{array}$ & $\begin{array}{l}\text { Rev. de } \\
\text { Enfermagem } \\
\text { UFPE online }\end{array}$ & Sul & Enfermeira \\
\hline 2017 & $\begin{array}{c}\text { 0Significados do } \\
\text { câncer de mama para } \\
\text { mulheres no contexto } \\
\text { do tratamento } \\
\text { quimioterápico }\end{array}$ & $\begin{array}{c}\text { MACHADO, } \\
\text { M.X; SOARES, } \\
\text { D.A; } \\
\text { OLIVEIRA, S.B }\end{array}$ & $\begin{array}{l}\text { Revista de } \\
\text { Saúde } \\
\text { Coletiva }\end{array}$ & Nordeste & Enfermeira \\
\hline 2018 & $\begin{array}{l}\text { Qualidade de Vida } \\
\text { Relacionada à Saúde } \\
\text { e Espiritualidade em } \\
\text { Pessoas com Câncer }\end{array}$ & $\begin{array}{l}\text { MENEZES, } \\
\text { R.R.; } \\
\text { et al. }\end{array}$ & $\begin{array}{c}\text { Revista } \\
\text { Brasileira de } \\
\text { Cancerologi } \\
\text { a }\end{array}$ & Nordeste & $\begin{array}{c}\text { Graduanda } \\
\text { em } \\
\text { Enfermagem }\end{array}$ \\
\hline 2018 & $\begin{array}{l}\text { "De corpo e alma": } \\
\text { histórias de mulheres } \\
\text { acometidas por câncer }\end{array}$ & $\begin{array}{c}\text { PESSÔA, M.S. } \\
\text { M. } \\
\text { et al. }\end{array}$ & $\begin{array}{c}\text { Rev. de } \\
\text { Enfermagem } \\
\text { online UFPE }\end{array}$ & Nordeste & Enfermeira \\
\hline 2019 & $\begin{array}{l}\text { Religião/espiritualidade } \\
\text { e apoio social na } \\
\text { melhoria da qualidade } \\
\text { de vida da pessoa com } \\
\text { cancro avançado }\end{array}$ & $\begin{array}{c}\text { SILVA, L.S et } \\
\text { al. }\end{array}$ & $\begin{array}{l}\text { Revista de } \\
\text { Enfermagem } \\
\text { Referência }\end{array}$ & Sul & Enfermeiro \\
\hline
\end{tabular}




\begin{tabular}{|c|c|c|c|c|c|}
\hline Ano & Título do artigo & Autores & Revista & Região & $\begin{array}{c}\text { Categoria } \\
\text { Profissional } \\
\text { do primeiro } \\
\text { autor }\end{array}$ \\
\hline 2019 & $\begin{array}{l}\text { Espiritualidade e } \\
\text { religião como recursos } \\
\text { para o enfrentamento } \\
\text { do câncer de mama }\end{array}$ & $\begin{array}{l}\text { RIBEIRO, } \\
\text { G.S.; } \\
\text { CAMPOS, } \\
\text { C.S.; ANJOS, } \\
\text { A.C. }\end{array}$ & $\begin{array}{l}\text { Rev. Fun. } \\
\text { Care Online }\end{array}$ & Sudeste & $\begin{array}{l}\text { Graduanda } \\
\text { em } \\
\text { Enfermagem }\end{array}$ \\
\hline 2019 & $\begin{array}{c}\text { Esperança de } \\
\text { mulheres em } \\
\text { tratamento } \\
\text { quimioterápico para } \\
\text { câncer de mama }\end{array}$ & $\begin{array}{c}\text { MACÊDO, } \\
\text { E.L.; GOMES, } \\
\text { E.T.; } \\
\text { BEZERRA, } \\
\text { S.M.M.S. }\end{array}$ & $\begin{array}{c}\text { Cogitare } \\
\text { Enfermagem }\end{array}$ & Nordeste & Enfermeiro \\
\hline 2019 & $\begin{array}{l}\text { Câncer de mama e } \\
\text { imagem corporal: } \\
\text { impacto dos } \\
\text { tratamentos no olhar } \\
\text { de mulheres } \\
\text { mastectomizadas }\end{array}$ & $\begin{array}{l}\text { OLIVEIRA, } \\
\text { T.B. } \\
\text { et al. }\end{array}$ & $\begin{array}{l}\text { Saúde e } \\
\text { Pesquisa }\end{array}$ & sudeste & $\begin{array}{l}\text { Graduando } \\
\text { em } \\
\text { Fisioterapia }\end{array}$ \\
\hline 2020 & $\begin{array}{c}\text { Estratégias de } \\
\text { enfrentamento após o } \\
\text { diagnóstico de câncer } \\
\text { de mama }\end{array}$ & $\begin{array}{c}\text { SILVA, K.K. et } \\
\text { al. }\end{array}$ & $\begin{array}{c}\text { Rev. } \\
\text { Brasileira } \\
\text { em } \\
\text { Promoção } \\
\text { da Saúde }\end{array}$ & Nordeste & Enfermeira \\
\hline 2020 & $\begin{array}{l}\text { Enfrentamento de } \\
\text { mulheres diante do } \\
\text { tratamento oncológico } \\
\text { e da mastectomia } \\
\text { como repercussão do } \\
\text { câncer de mama }\end{array}$ & $\begin{array}{l}\text { SILVA F.C.N.; } \\
\text { ARBOIT, E.L.; } \\
\text { MENEZES, } \\
\text { L.P. }\end{array}$ & $\begin{array}{l}\text { Rev. Online: } \\
\text { Cuidado } \\
\text { Fundamenta } \\
\text { I }\end{array}$ & Sul & Enfermeira \\
\hline
\end{tabular}

Para melhor expor, a análise, interpretação e discussão dos artigos, optou-se pela divisão em categorias, sendo elas: A espiritualidade no enfrentamento do câncer de mama: aceitação, resiliência e conforto; $A$ rede de apoio social e a religiosidade/espiritualidade no enfrentamento do Câncer de mama; e A dimensão espiritual como instrumento de prática profissional no cuidado holístico a mulheres com câncer de mama. Que serão apresentadas a seguir. 
A influência da espiritualidade/religiosidade no enfrentamento do câncer de mama: uma revisão integrativa Araújo MN, Araújo GSF, Costa M.M., Espírito Santo C.C., Pontes APM

\section{A espiritualidade no enfrentamento do câncer de mama: Aceitação, Resiliência e Conforto}

Descobrir um câncer gera sentimentos extremos, em sua maioria negativos, como a sensação iminente de morte e de perda, o que muitas vezes acarreta medo do desconhecido. A partir desta revisão foi possível perceber que a dimensão espiritual possui um papel protagonista no processo de enfrentamento do câncer de mama, uma vez que funciona como uma fonte inesgotável de conforto, auxilia no processo de aceitação e de resiliência.

Silva, Arboit, Menezes (2020) referem que a família é indispensável para o tratamento fluir da melhor maneira possível, eles se apegam na fé em busca da esperança para alcançar a cura da doença. Fato este que também foi identificado no estudo de Santos et al. (2017, p.3225) ao identificarem que além do suporte familiar, "a Fé em Deus, a crença no Ser Superior, é fonte de suporte e conforto para enfrentar o tratamento e a doença".

Ao lidar com o processo de adoecimento essas mulheres buscam diferentes formas de apoio, dentre eles, "a fé e otimismo são ferramentas de enfrentamento fundamentais em situações difíceis ocasionadas pelo câncer, pois proporcionam esperança e força, permitindo que as mesmas lidem de maneira diferente com a doença” (OLIVEIRA et al. 2019).

Segundo Pessôa et al. (2018), a espiritualidade quebra o estigma da doença câncer fazendo com que a mulher a enfrente e aceite com maior naturalidade, como enfrentaria outra patologia que porventura venha a afligir.

Corroborando com Pessôa et al. (2018), Ribeiro, Campos e Anjos (2019) afirmam que ao enfrentar e aceitar a trajetória do câncer a espiritualidade/religiosidade confortam e impulsionam sentimentos positivos. Menezes et al. (2018) também abordam essa aceitação proporcionada pela espiritualidade/religiosidade, quebrando paradigmas da finitude que a doença traz, ressignificando a vida positivamente.

O poder que a espiritualidade exerce na vida das mulheres é notório nos estudos selecionados. Santos et al. (2017) afirmam que a fé em Deus promove 
A influência da espiritualidade/religiosidade no enfrentamento do câncer de mama: uma revisão integrativa Araújo MN, Araújo GSF, Costa M.M., Espírito Santo C.C., Pontes APM

a renovação de forças para que o processo seja encarado como uma luta mais amena, alicerçada pela fé. Para Silva et al. (2019) a espiritualidade/religiosidade remete à transcendência, ao conforto diante do corpo mutilado e da queda do cabelo, sendo assim, a espiritualidade melhora qualidade de vida.

\section{A rede de apoio social e a religiosidade/espiritualidade no enfrentamento do câncer de mama}

O impacto causado pelo diagnóstico do câncer traz várias fragilidades da mulher à tona, o meio em que ela vive irá influenciar de forma intensiva no seu tratamento. Sá apud Oliveira et al. (2019) refere que a abstinência laboral e inversão de papeis da mulher antes cuidadora agora com objeto de cuidado aflora ainda mais os sentimentos negativos. Em seu estudo, Oliveira et al. (2019) discorre sobre a compreensão que a família deve ter. Para Santos et al. (2017), a rede apoio formada por familiares e amigos humaniza o processo de enfretamento.

Silva et al. (2020) aponta que o cônjuge possui um papel especial, uma vez que tem papel crucial na vida dessa mulher muitas vezes mutilada, que necessita se redescobrir num corpo "novo". Santos et al. (2017) corrobora com este achado ao afirmar que 0 marido possui um papel crucial para 0 enfrentamento das dificuldades encontradas durante o tratamento. Macêdo, Gomes e Bezerra (2019) identificaram em seu estudo que mulheres com companheiros apresentaram maior índice de esperança no tratamento, o que caracteriza a presença e apoio do companheiro como fundamental.

"A doença traz a oportunidade de reavaliar sentimentos e posturas dentro da relação e, desse modo, a recuperação da mulher fica condicionada à forma como o parceiro aceita a situação" (SILVA et al.,2020, p.5). "[...] O afeto familiar direciona uma melhor estabilidade emocional e se constitui ponto de apoio na luta contra a doença, porque a mulher possuirá relativa aceitação do diagnóstico [...]" (NASCIMENTO et al., 2015, p. 112) 
A influência da espiritualidade/religiosidade no enfrentamento do câncer de mama: uma revisão integrativa Araújo MN, Araújo GSF, Costa M.M., Espírito Santo C.C., Pontes APM

Dessa forma, também é possível perceber 0 apoio da espiritualidade/religiosidade no estudo de Silva et al. (2020), onde as mulheres relatam em suas falas que o apoio recebido pela comunidade religiosa ajuda no enfrentamento do câncer de mama, os autores também relatam que esse apoio ofertado pela religião/ espiritualidade ajudam a essas mulheres transcenderem e a enfrentarem a doença de forma mais positiva.

Corroborando com este achado, o estudo de Silva et al. (2019) refere que a maioria dessas mulheres faz uso dessa estratégia como forma de apoio, seja por meio de visitas ou orações da comunidade religiosa, e que a mesma contribui para a melhora da qualidade de vida, promove maior adesão ao tratamento e maior bem estar emocional/espiritual.

Por fim, Ribeiro, Campos e Anjos (2019) referem que a ida em centros religiosos proporciona maior conforto a esta mulher, sendo uma importante rede de apoio e auxiliando no enfrentamento desta nova condição. Santos et al. (2017) identificaram em seu estudo que Deus, o templo religioso ou alguma forma de divindade é primeiro ponto de apoio que as mulheres encontram para poder enfrentar o câncer.

\section{A dimensão espiritual como instrumento de prática profissional no cuidado holístico a mulheres com câncer de mama}

Diversos artigos analisados abordaram a importância dos profissionais de saúde, em especial da enfermagem, de valorizar a dimensão espiritual das mulheres diagnosticadas com câncer de mama. Ribeiro, Campos e Anjos (2019) afirmam que profissionais de saúde que possuem maior compreensão acerca da espiritualidade/religiosidade dos pacientes conseguem aprimorar sua avaliação e prática clínica, além de melhorar sua relação com paciente e familiares/cuidadores, oferecendo apoio às suas práticas religiosas e espirituais, o que auxilia no enfrentamento da doença.

Machado, Soares e Oliveira (2017) abordam a escuta ativa que o profissional deve dispensar a essa mulher, tornando-a autônoma no tratamento 
e auxiliando a quebrar barreiras. Já Nascimento et al. (2015) contribuíram com esta análise ao afirmarem que o enfermeiro é o provedor da educação em saúde e potencializador do autocuidado. Assim, a escuta ativa é essencial para a identificação das necessidades espirituais da mulher com câncer de mama.

Outro papel crucial que a enfermagem desempenha é o alívio dos sintomas de dor e angústia diante do inesperado. "A relação de confiança da enfermagem com as pacientes possibilitou a realização de uma assistência qualificada, promovendo suporte físico, emocional e psicológico" (SILVA; ARBOIT; MENEZES, 2020, p.362). Inclui-se igualmente nesta assistência o apoio espiritual que ameniza a angústia e dá sentido à experiência vivida, conforme discutido na categoria anterior.

Pêssoa et al. (2018) corroboram com a reflexão ao discutir o papel do profissional em seu estudo e referem que uma vez que o profissional perde o olhar humanizado e volta-se somente para a patologia, deixa de enxergar singularidade de uma mulher que perdeu algo muito importante que está ligado tão intimamente a feminilidade, o que torna o tratamento mais penoso (PESSÔA et al., 2018).

Ribeiro, Campos e Anjos (2019) falam da importância de os profissionais abordarem a dimensão espiritual no manejo do câncer, discutem sobre a importância deste elemento estar presente desde a formação profissional, de forma que esta aborde o holismo, o olhar o ser um humano como um todo, desde o começo da graduação.

\section{CONCLUSÃO}

Com base na análise realizada foi possível concluir que a espiritualidade/religiosidade representada por Deus e pela fé se caracterizam como uma estratégia importante no enfrentamento do câncer de mama, promovendo mais conforto e alívio dos impactos causados pela doença, além de bem-estar e resiliência. 
Foi possível identificar diversos estudos que apontam a espiritualidade como suporte fundamental para o enfrentamento do câncer de mama, ressignificando o sentido da vida, sendo uma fonte de apoio e de força, favorecendo adesão ao tratamento e a vivência do tratamento de forma mais esperançosa.

A espiritualidade/religiosidade como uma dimensão da qualidade de vida apresenta-se como uma experiência importante na vida da mulher com diagnóstico de câncer de mama, uma vez que auxilia no enfrentamento do processo, juntamente com seus familiares que se tornam essenciais nesse momento. Neste sentido, uma rede de apoio sólida se caracteriza como fundamental, composta por família, amigos e locais de vivência e expressão da espiritualidade/religiosidade.

Foi possível identificar que os elementos da tríade: vivência da espiritualidade, rede de apoio social e atuação da equipe de saúde são percebidos como alicerces para o enfrentamento do câncer de mama. Sendo assim, conhecer e valorizar a vivência espiritual dessas pacientes torna-se, então, instrumento de grande valor, inclusive pelos profissionais que prestam cuidados diretamente a elas, uma vez que, contribuem para o bem-estar e sensação de apoio e assistência.

Também foi possível identificar, nos estudos selecionados, que valorizar a vivência da espiritualidade/religiosidade é uma estratégia essencial no cuidado a essas mulheres, e que a atuação multiprofissional e a capacitação dos profissionais para este olhar são pontos fundamentais, bem como compreender a percepção dos mesmos sobre este processo, abrindo a seguinte lacuna para futuros estudos: como os profissionais de saúde enxergam a influência da espiritualidade/religiosidade na assistência aos pacientes com câncer de mama?

Destaca-se como limitação deste estudo a busca de artigos apenas em língua portuguesa, considerando-se que a busca em outras línguas poderá ampliar a contextualização dos resultados. 
A influência da espiritualidade/religiosidade no enfrentamento do câncer de mama: uma revisão integrativa Araújo MN, Araújo GSF, Costa M.M., Espírito Santo C.C., Pontes APM

\section{REFERÊNCIAS}

AITKEN, E.V.P. O papel do assistente espiritual na equipe. In: CARVALHO, R.T.; PARSONS, H.A. (org.). Manual de Cuidados Paliativos. 2. ed. São Paulo: ANCP - Academia Nacional de Cuidados Paliativos, 2012, p. 364-365.

CAETANO, J.A., SOARES E. - Mulheres mastectomizadas diante do processo de adaptação de self-físico e self-pessoal. Revista de Enfermagem da UERJ, v.13, n.2, p.201-16, 2005.

INSTITUTO NACIONAL DO CÂNCER - INCA, 2019. Disponível em: www.inca.gov.br/. Acesso em: 08 mar 2020

MACÊDO, E.L.; GOMES, E.T.; BEZERRA, S.M.M.S. Esperança de mulheres em tratamento quimioterápico para o câncer de mama. Cogitare Enfermagem, v. 24, p. e65400, 2019, disponível em: http://dx.doi.org/10.5380/ce.v24i0.65400. Acesso em: 11 out 2020.

MACHADO, M.X.; SOARES, D.A.; OLIVEIRA, S.B. Significados do câncer de mama para mulheres no contexto do tratamento quimioterápico. Physis: Revista de Saúde Coletiva, v. 27, p. 433-451, 2017.

MENDES, K. das S.; SILVEIRA, R.C.C.P.; GALVAO, C.M. Revisão integrativa: método de pesquisa para a incorporação de evidências na saúde e na enfermagem. Texto \& contexto enfermagem, v. 17, n. 4, p. 758-764, 2008. Disponível em: http://www.scielo.br/scielo.php?script=sci_arttext\&pid=S010407072008000400018\&lng=en\&nrm=iso. Acesso em: 17 Nov 2020.

MENEZES, R.R. et al.. Qualidade de vida relacionada à saúde e espiritualidade em pessoas com câncer. Revista Brasileira de Cancerologia, v. 64, n. 1, p. 917, 2018.

MESQUITA, A.C. et al. A utilização do enfrentamento religioso/espiritual por pacientes com câncer em tratamento quimioterápico. Revista Latino Americana de Enfermagem [Internet], v. 21, n. 2, mar./abr., 2013. Disponível em: https://www.scielo.br/pdf/rlae/v21n2/pt_0104-1169-rlae-21-02-0539.pdf acesso: 15 jun. 2020.

NASCIMENTO, K.T.S. et al. Sentimentos e fontes de apoio emocional de mulheres em pré-operatório de mastectomia em um hospital-escola. Revista Enfermagem, v.23 n.1 p. 108-114, 2015.

OLIVEIRA, T.B; et al. Câncer de mama e imagem corporal: impacto dos tratamentos no olhar de mulheres mastectomizadas. Saúde e Pesquisa, v. 12, n. 3 , p. $451-462,2019$ 
PESSÔA, M.S.M. et al. " De corpo e alma": histórias de mulheres acometidas por câncer. Revista de Enfermagem online UFPE, v.12, n.3, p.642-650, 2018.

RIBEIRO, G.S.; CAMPOS, C.S.; ANJOS, A.C. Espiritualidade e religião como recursos para o enfrentamento do câncer de mama. Revista on line de pesquisa cuidado fundamental., v.11, p. 849-856, 2019. Disponível em: http://dx.doi.org/10.9789/2175- 5361.2019.v11i4.849-856. Acesso em: 12 out. 2020.

SAPORETTI, L.A.; SILVA, A.M.O.P. Aspectos particulares e ritos de passagem nas diferentes religiões. In: CARVALHO, R.T.; PARSONS, H.A. (org.). Manual de Cuidados Paliativos. 2. ed. São Paulo: ANCP - Academia Nacional de Cuidados Paliativos, 2012, p. 556-568.

SANTOS, I.D.L, et.al. Câncer de mama: o apoio recebido no enfrentamento da doença. Revista enfermagem UFPE on line, v.11, p. 3222-3227, 2017.

SILVA, F.C.N.; ARBOIT, E.L.; MENEZES, L.P. Enfrentamento de mulheres diante do tratamento oncológico e da mastectomia como repercussão do câncer de mama. Revista Online: Cuidado Fundamental, v.12 p.357-363,2020.

SILVA, K.K. et al. Estratégias de enfrentamento após o diagnóstico de câncer de mama. Revista Brasileira em Promoção da Saúde, v.33, p.1-9, 2020. Disponível em: https://periodicos.unifor.br/RBPS/article/view/10022/pdf. Acesso em: 15 nov. 2020.

SILVA, L.S. et al. Religião/espiritualidade e apoio social na melhoria da qualidade de vida da pessoa com cancro avançado. Referência, v. 4, n. 23, p. 111-120, 2019

SOUZA, M.T de; SILVA, M.D. da; CARVALHO, R. de. Revisão integrativa: o que é e como fazer. Einstein (São Paulo), São Paulo, v.8, n.1, p.102-106, 2010. Available from <http://www.scielo.br/scielo.php?script=sci_arttext\&pid=S167945082010000100102\&lng=en\&nrm=iso>. Acesso em: 17 Nov. 2020. 